\title{
Considerations of Joule heating and radius reduction in umbral dots
}

\author{
H. Hamedivafa ${ }^{\star}$ \\ Department of Physics, Sharif University of Technology, PO Box 11365-9161, Tehran, Iran \\ Received 14 November 2002 / Accepted 26 Mars 2003

\begin{abstract}
The physical nature of bright features in sunspot umbra such as umbral dots (UDs) is not fully understood yet. Here, by accepting magnetic field reduction in the UDs, the Joule heating mechanism in the UDs is investigated. In this investigation, a UD model is assumed which is characterized by a) a reduced (possibly zero) magnetic field strength in the UD column relative to its surroundings, b) the electric current generated by magnetic field gradient at the boundary of the UD column can produce some heat, and c) when the radius of the UD - because of pressure reduction inside the column - becomes zero, the death of the UD will occur. The rate of radius reduction is estimated to be nearly $100 \mathrm{~m} \mathrm{~s}^{-1}$. Without consideration of gas injection from the bottom of the UD column, it is found that the speed of outflow in UDs varies linearly from zero at the bottom to a few $100 \mathrm{~m} \mathrm{~s}^{-1}$ at the upper level. It is shown that Joule heating, in some cases, can produce enough heat to explain the brightness of UDs.
\end{abstract}

Key words. Sun: sunspots - Sun: magnetic field

\section{Introduction}

One of the bright features (see Sobotka et al. 1993, for a review) which is seen in sunspot umbra is the umbral dot (UD). From the observational point of view, the most important physical parameters of UDs are their brightness, lifetime, magnetic field, size, mass outflows (upward motion), and filling factor. Some observational reports about these parameters are summarized here.

Sobotka et al. (1997a, 1997b) have shown that there is no "typical" lifetime and size for the UDs - the number of UDs increases by decreasing their lifetime and size (diameter). However, the average diameter of UDs is less than $300 \mathrm{~km}$ (Beckers \& Schröter 1968; Koutchmy \& Adjabshirzadeh 1981; Lites et al. 1991; Tritschler \& Schmidt 2002). Ewell (1992) and Sobotka (1997a) have reported a lifetime about 15 min in average. It has been observed that several UDs have lifetimes of more than 2 h (Kusoffosky \& Lundstedt 1986; Ewell 1992; Sobotka et al. 1997a).

Although, there are observations reporting no drastic magnetic field reduction in the UDs (Lites et al. 1991), many spectroscopic observations indicate that the UDs have a much weaker magnetic field strength than their surrounding umbra. Buurman (1973), Adjabshirzadeh \& Koutchmy (1983) found a field strength in the UDs, about $10 \%$ smaller relative to the surrounding umbra. A reduced field about 20-30\% has been reported by Pahlke \& Wiehr (1990), Kneer et al. (1990) have measured a $15 \%$ weaker magnetic field, and

\footnotetext{
* e-mail: vafa@mehr.sharif.edu
}

Wiehr \& Degenhardt (1993) have found a field about $20 \%$ smaller in the UDs relative to the surrounding umbra.

Also, on the basis of spectroscopic observations, a mass upward motion in the UDs has been observed. Some of these observations show that the outflow speed in the UDs is not greater than a few hundred $\mathrm{m} \mathrm{s}^{-1}$ (Lites et al. 1991; Wiehr 1994; Rimmele 1997). Pahlke \& Wiehr (1990) inferred speed of $1.2 \mathrm{~km} \mathrm{~s}^{-1}$ for the outflows.

Many reports give a relative intensity of the UDs and their surroundings. The value of $\frac{I_{\mathrm{UD}}}{I_{\mathrm{u}}}-$ in which $I_{\mathrm{UD}}$ and $I_{\mathrm{u}}$ are the intensities of the UD and its surrounding umbra, respectively - varies from 1.1 (Grossmann-Doerth et al. 1986; Sobotka et al. 1992a) to 2.6 (Sobotka et al. 1992b). Recently, Tritschler $\&$ Schmidt (2002) have measured the average temperature of the UDs to be $37 \%(330 \mathrm{~K})$ brighter than the mean intensity of the surrounding umbra. The histogram of the UD brightness shows two peaks which may correspond to two populations and also to two mechanisms of creation or evolution of the UDs (Tritschler \& Schmidt 2002; Sobotka et al. 1997b).

The spatial distribution of the UDs is nonuniform and their density increases with increasing mean intensity of their background. At any instant, they occupy less than $15 \%$ of the total area of the umbra (Bumba \& Suda 1980; Sobotka et al. 1997a; Tritschler \& Schmidt 2002).

The physical nature of the UDs (or any other fine structure in sunspots) has not been fully understood yet. In the next section, some aspects of the models of Parker/Choudhuri and Degenhardt \& Lites - which want to explain the creation or existence of the UDs and/or their characteristics - have been compared. In Sect. 3, by some assumptions about the magnetic 
field in a typical UD, it has been shown that - because of the lateral pressure gradient at the UD's boundaries - the radius of the UD column becomes smaller and smaller during its lifetime, until it becomes zero (and finally disappears). Joule heating in the UDs can produce some heat which may explain the brightness of the UDs. This mechanism is discussed in Sects. 4 and 5. Hirayama (1992) has investigated Joule heating power generated in a slender magnetic flux tube in the upper photospheric layers. He has shown that this mechanism may supply enough energy to heat the temperature minimum region of the flux tube (excess energy flux of faculae, spicule formation).

\section{Comparison of UD models}

In Parker's (1979a) sunspot model, the region below the visible surface of the umbra is made up of individual flux tubes embedded in a field-free plasma. At the visible surface, the upper flaring part of the magnetic field is composed by combination of these flux tubes into a single flux tube - so, a spot forms. Parker conjectured that the separated tubes have diameters less than $300 \mathrm{~km}$. He argued that the depth at which the tube separation occurs is probably not less than $300 \mathrm{~km}$ and not more than a few thousand $\mathrm{km}$. Also, he postulated a strong convective downdraught in the field-free gas between the individual flux tubes underneath the sunspot. This idea seems to be confirmed by Zhao \& Kosovichev (2001). They found a powerful converging and downward flow at depths of 1.5-5.0 Mm.

Parker (1979b) calculated the overstable modes for a vertical slab of a field-free gas (fluid) sandwiched between two regions of a vertical magnetic field. He found that if the fieldfree fluid is convectively unstable, vertical oscillations of period $100 \mathrm{~s}$ with a vertical wavelength of $1000 \mathrm{~km}$ can be stimulated in it. The growth time scale of these oscillations is $1-3 \mathrm{~h}$. He proposed that a UD is an intrusion of a field-free fluid because of the vertical oscillations - from somewhere beneath the sunspot through the flux tubes towards the surface.

The static configuration of a field-free gas column (packet) trapped by a magnetic field has been obtained by Choudhuri (1986) who showed that it has a tapering shape with a certain apex. He also concluded that if the pressure of the field-free gas increases, the apex of the packet rises higher and higher until it reaches a certain height where the magnetic pressure suddenly becomes negligible above it. Ultimately, the trapped gas (closed packet) bursts and a (large) amount of field-free gas escapes at a speed of the order of the Alfvén speed $\left(10 \mathrm{~km} \mathrm{~s}^{-1}\right.$ in the surrounding magnetized gas) and then, a UD is created. By comparing the mean free paths of photons at different depths, he tried to explain that the heat carried by convection at a depth of $5000 \mathrm{~km}$ below the photosphere may pile up and cause the packet to rise. He has given a rough estimation for the ascent speed of the apex of the gas packet. This is a very low speed, nearly $0.03 \mathrm{~km} \mathrm{~s}^{-1}$ at depth of $1000 \mathrm{~km}$. However, on the basis of this argument and knowing the limited transverse dimension of a UD, it seems that the radiation diffusion should be very large in the upper layers. Parker (1979b) also found that a column of gas with a sufficient diameter $(\geq 100 \mathrm{~km})$ rising at a sufficient rate $\left(\geq 0.5 \mathrm{~km} \mathrm{~s}^{-1}\right)$ remains significantly warmer than its surroundings. Thus, it seems that at depths of a few thousand $\mathrm{km}$, a hot gas packet with a low speed cannot be retained for longer than a few seconds.

Parker (1979b) believes that the lifetime of continued intrusion of hot gases from the field-free column (the lifetime of a UD) is related to the 1-3 $\mathrm{h}$ growth time of the stable oscillation modes stimulated in the field-free gas beneath the sunspot. However, it seems that (at first) the rising time of a trapped field-free gas (gas packet) is related to the 1-3 h growth time of these oscillations. If so, the hot gas packet has cooled before it reaching the surface layers because the rising time is very long.

In the model of Degenhardt \& Lites (1993) - a magnetohydrodynamical model - UDs are considered as thin vertical magnetic flux tubes with a reduced (but nonzero) magnetic field strength, a temperature enhancement and a material upward flow at the bottom, embedded in a stationary sunspot umbra. They showed that under some boundary conditions, the magnetohydrodynamical calculations of the evolution of these flux tubes lead to a steady material outflow. Then some bright points relative to the surrounding umbra might form.

In this model some constraints and boundary conditions have been considered:

1. the stationary state of the outflow (Eqs. (1) and (2) in Degenhardt \& Lites 1993),

2. the balance condition of the lateral forces at all heights (Eq. (8) in Degenhardt \& Lites 1993),

3. the conservation condition of the magnetic flux in the UD flux tube and that in the surrounding region of the UD flux tube which is limited to a cylindrical surface with constant radius (Eqs. (13)-(16) in Degenhardt \& Lites 1993).

Satisfying these constraints predicts necessarily a special geometrical shape for the UD flux tube. It is worthwhile to note that before the material outflow reaches a steady state with nonzero velocity, a closed packet of hot gases has existed in a dynamical equilibrium with its surroundings and without a considerable material flow within it. In these conditions only constraints (2) and (3) may determine the geometrical shape of the gas packet (Choudhuri 1986) and constraint (1) with assumption of nonzero flow velocity is not true. After reaching the surface, a "magnetic valve" (as proposed by Choudhuri 1986) would be opened and lead to the intrusion of some hot gases at the order of the Alfvén speed $\left(10 \mathrm{~km} \mathrm{~s}^{-1}\right)$ in the surroundings. Thus, if no more gas is injected into the flux tube, a significant reduction of pressure and density occurs quickly inside the UD flux tube. In this situation, radiative dissipation from the hot gas column also becomes more efficient and the temperature decreases quickly. Thus, lateral forces do not balance (violation of constraint (2)), and so the radius of the UD flux tube becomes smaller and smaller at all heights along the flux tube length. As a result of the radius reduction, the material outflows can hold the "magnetic valve" open for a certain time interval. In this situation, the constraint (3) no longer can be satisfied. So, conservation of magnetic flux may only be considered inside the flux tube.

Also, Choudhuri (1986) has given a qualitative description of the steady state flow for an incompressible fluid in flux tube 

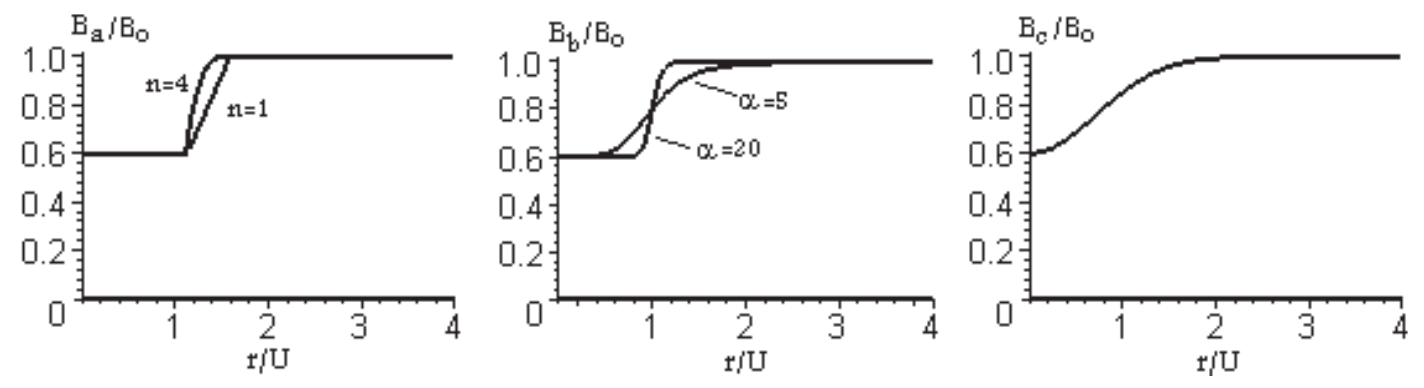

Fig. 1. The profiles of UD magnetic field according to relations ( $1 \mathrm{a}, \mathrm{b}, \mathrm{c})$ for $\gamma=0.4$.

on the basis of these three constraints. It seems that a real dynamical model can be obtained by considering the radius reduction of the UD tube.

In this paper it is proposed that the vertical oscillations of the hot field-free fluid which were proposed by Parker (1979b) - or non-linear magnetoconvection (Knobloch \& Weiss 1984) as examples of possible mechanisms - may produce only the "seed" of a UD. This means that possibly the vertical oscillations may grow to large amplitudes to eject quickly a pocket of hot fluid into the visible region of the umbra. Then a (large) amount of the fluid can escape and thereby a column will form with less density and weaker (very much weaker) magnetic field strength relative to the surroundings.

In the following a model is given in which the radius reduction of the UD flux tube (gas column) has been considered. Joule heating mechanism, Sects. 4 and 5, can generate some heat at the boundary of UD column where the gradient of the magnetic field is considerable. The generated heat, in some cases, can explain the brightness of the UDs.

\section{Theoretical model}

The UD model which is investigated here is characterized by:

1. a reduced (or zero) magnetic field strength in the UD column relative to the surrounding umbra,

2. an electric current generated by the magnetic field gradient at the boundary of the UD column, which can produce some energy,

3. the death of a UD will occur when it collapses and the radius of its column becomes zero (at extreme).

\subsection{Magnetic field in UDs}

For simplicity we assume that the magnetic field vector has only a vertical component and that its magnitude is only a function of distance from the axis of the UD column

$\boldsymbol{B}=B(r) \hat{k}$

where $\hat{k}$ is unit vector along $z$-axis and $r$ is the radial component in cylindrical coordinates.

According to the reports on the magnetic field reduction in the UDs, one can say that this reduction should not be observed beyond the observed radius of a bright UD. Therefore, one of the following forms of $B(r)$ may be considered:

a) $B_{\mathrm{a}}(r)= \begin{cases}(1-\gamma) B_{0} & r \leq U(t) \\ B_{0}\left(1-\gamma\left(\frac{R(t)-r}{R(t)-U(t)}\right)^{n}\right) & U \leq r \leq R \\ B_{0} & R(t) \leq r\end{cases}$

b) $B_{\mathrm{b}}(r)=B_{0}-\frac{\gamma B_{0}}{1+\left(\frac{r}{U(t)}\right)^{\alpha}}$

c) $B_{\mathrm{c}}(r)=B_{0}-\gamma B_{0} \exp \left(-\frac{r^{2}}{U^{2}(t)}\right)$,

where $r$ is the distance from the UD axis, $U(t)$ is the radius of the UD column at any instant, $B_{0}$ is the undisturbed magnetic field strength of the umbra, and $0 \leq \gamma \leq 1$ is the fraction of the field strength reduction on the central axis of the UD column. The upper and lower limits of $\gamma$ are related to zero field strength and field strength of $B_{0}$ at the center of the UD column, respectively. The parameters $n$, in Eq. (1a), and $\alpha$, in Eq. (1b), are free parameters which determine the field gradient around the radius of the UD column. $R(t)(>U(t))$ in relation (1a) is also a measure of the instantaneous radius of the UD column. In relation (1a), at distances $U(t)<r<R(t)$, gradient of the field is not zero and $B(r)$ increases from $(1-\gamma) B_{0}$ to $B_{0}$. Figure 1 shows the profiles of these three forms of $B(r)$ for the same $\gamma$ values.

Gokhale \& Zwaan (1972) and Petrovay et al. (1999) have used similar $B(r)$ profiles of (1a) and (1b), respectively, to describe the sunspot decay.

\subsection{Considerations of radius reduction}

In order to present a simple theoretical model, we use a magnetic field defined by the relation (1b). The magnetic flux, $\phi(r, t)$, through a circle, $C(r)$, whose radius is $r$ at a constant $z$ can be obtained as

$$
\begin{aligned}
\phi(r, t) & =\int_{0}^{r} B(r)(2 \pi r) \mathrm{d} r \\
& =\pi B_{0} U^{2}(t)\left(q^{2}-\gamma I\right),
\end{aligned}
$$

where $q=\frac{r}{U}$ is the normalized radial distance and $I$ is defined as

$I=2 \int_{0}^{r / U} \frac{\omega \mathrm{d} \omega}{1+\omega^{\alpha}}$ 
The rate of change of the magnetic flux through the circle $C(r)$ ( $r$ is a constant parameter), can be computed simply from Eq. (2) as

$$
\frac{\partial \phi}{\partial t}=-2 \pi B_{0} U \dot{U}\left(\gamma I-\frac{\gamma q^{2}}{1+q^{\alpha}}\right)
$$

where $\dot{U}=\frac{\mathrm{d} U}{\mathrm{~d} t}$. In this derivation, it is assumed that $\alpha$ is time independent. One can define a measure of normalized thickness $k$, of the region in which the magnetic field gradient is considerable, as (see Fig. 1)

$k=\frac{\gamma B_{0}}{U(\mathrm{~d} B / \mathrm{d} r)_{r=U}}=\frac{4}{\alpha}$.

The time independence of $\alpha$ means that the normalized thickness, $k$, is time independent. A radial component in the velocity field of the fluid may guarantee this assumption (see below).

On the other hand, by integrating the $z$-component of the equation

$$
\frac{\partial \boldsymbol{B}}{\partial t}=\boldsymbol{\nabla} \times(\boldsymbol{v} \times \boldsymbol{B}-\eta \boldsymbol{\nabla} \times \boldsymbol{B})
$$

over the area of the circle $C(r)$ defined above, the rate of change of the magnetic flux through circle $C(r)$ can be computed as

$$
\frac{\partial \phi(r, t)}{\partial t}=2 \pi r\left((\boldsymbol{v} \times \boldsymbol{B})_{\varphi}-\eta(\boldsymbol{\nabla} \times \boldsymbol{B})_{\varphi}\right),
$$

where the $\varphi$-index indicates the azimuthal component in cylindrical coordinate and $\eta$ is the electrical resistivity, given by $\eta=\frac{c^{2}}{4 \pi \sigma}$ (cgs), in which $\sigma$ is the electrical conductivity. Since, $\boldsymbol{B}=B(r) \hat{k}$, Eq. (6) is simplified to

$$
\frac{\partial \phi(r, t)}{\partial t}=2 \pi r\left(-v_{\mathrm{r}} B+\eta \frac{\partial B}{\partial r}\right)
$$

where $v_{\mathrm{r}}$ is the radial component of the velocity field.

Without a specific velocity field, Ohmic diffusion will reduce the field gradient. The nonzero radial velocity in Eq. (7) may retain the nonvanishing (possibly large) magnetic field gradient for a longer time comparing to the characteristic time of Ohmic diffusion.

By substituting $B(r)$ (the form of Eq. (1b)) into Eq. (7), we have in terms of $q$ :

$$
\frac{\partial \phi(r, t)}{\partial t}=2 \pi B_{0} q\left(-v_{\mathrm{r}} U\left[1-\frac{\gamma}{1+q^{\alpha}}\right]+\alpha \eta \gamma \frac{q^{\alpha-1}}{\left(1+q^{\alpha}\right)^{2}}\right) .
$$

Comparing Eqs. (3) and (8), we have

$$
\begin{aligned}
& -2 \pi B_{0} U \dot{U}\left(\gamma I-\frac{\gamma q^{2}}{1+q^{\alpha}}\right) \\
& =2 \pi B_{0} q\left(-v_{\mathrm{r}} U\left[1-\frac{\gamma}{1+q^{\alpha}}\right]+\alpha \eta \gamma \frac{q^{\alpha-1}}{\left(1+q^{\alpha}\right)^{2}}\right) .
\end{aligned}
$$

We want to calculate Eq. (9) at the defined boundary of the UD column - at the limit $r \rightarrow U$ (or $q \rightarrow 1$ ). At this limit, one can see that $0.6<I<1$ for $\alpha \geq 1$ as $\alpha$ gets larger the $I$ value gets closer to one. For $\alpha=1,10,20$, we have $I=0.614,0.888,0.938$, respectively. Therefore, for large $\alpha$ (see Sect. 4; thinness coefficient) $I \approx 1$.
Assuming that $\eta$ does not depend on $B$, then at the boundary of the UD column $(q \rightarrow 1)$ Eq. (9) yields

$$
-U \dot{U} \approx-v_{\mathrm{r}} U\left(\frac{2}{\gamma}-1\right)+\frac{\alpha \eta}{2} .
$$

Now Eq. (10) can be used for a typical value of the UD characteristics. Let us take the radius of the UD, $U=150 \mathrm{~km}$ and its lifetime, $\tau_{\mathrm{UD}}=15 \mathrm{~min}$ (Sobotka et al. 1997a). If we approximate the observed UD area, $A_{\mathrm{u}}$, by $\pi U^{2}$ and $\dot{A}_{\mathrm{u}}=\frac{\mathrm{d} A_{\mathrm{uD}}}{\mathrm{d} t}$ by $\left(-\frac{A_{\mathrm{UD}}}{\tau_{\mathrm{UD}}}\right)$, we obtain

$-U \dot{U} \approx \frac{A_{\mathrm{UD}}}{2 \pi \tau_{\mathrm{UD}}}=\frac{U^{2}}{2 \tau_{\mathrm{UD}}} \approx 1.25 \times 10^{11}\left(\mathrm{~cm}^{2} \mathrm{~s}^{-1}\right)$.

By assuming $\eta \approx 10^{9}$ (cgs) for the surface layers of the umbra, for large $\gamma$ values $(\gamma \approx 1)$, we find that the second term in the right hand side of Eq. (10) - for $\alpha<200$ - is not large enough to satisfy the equality. Then, the velocity field should have a negative radial component (an inward flow) with a magnitude of nearly $\dot{U}$ (nearly $v_{\mathrm{r}} \approx \dot{U} \approx-100 \mathrm{~m} \mathrm{~s}^{-1}$ ) at the boundary of UD column. Also, for small $\gamma$ values, a negative radial velocity in the order of $\dot{U}$ is not excluded.

It is obvious that the radial component of the total pressure gradient determines the radial velocity component. If the pressure reduction of the gas in a UD column is large, a large lateral flow can occur at all heights. Then, it may be possible for the lower part of the UD column to be closed sooner than the "magnetic valve" at its upper part. An exact result can be obtained by numerically solving a complete set of MHD equations.

Now, we can estimate the upward velocity of gas, $v_{\text {up }}$, in a UD. By some assumptions such as the incompressibility of the gas, constant temperature, and the lack of gas injection from the bottom of the UD column, the continuity of mass flow yields:

$\rho(2 \pi U \ell)\left|v_{\mathrm{r}}\right|=\rho\left(\pi U^{2}\right) v_{\mathrm{up}}$,

then,

$v_{\text {up }}=\left(\frac{2\left|v_{\mathrm{r}}\right|}{U}\right) \ell$

where the height, $\ell$, is measured from the bottom of the UD column and $v_{\text {up }}$ is the upward speed at height $\ell$ in the UD. If we accept that a UD has a length of about $300 \mathrm{~km}$ (see discussion below Eq. (22)), the upward speed will vary linearly from zero at the bottom to nearly $400 \mathrm{~m} \mathrm{~s}^{-1}$ at the top, taking $U=150 \mathrm{~km}$ and $\left|v_{\mathrm{r}}\right|=100 \mathrm{~m} \mathrm{~s}^{-1}$. With elapsing time, $U$ becomes smaller, thereby this maximum value may become larger.

\section{Joule heating power in a UD}

In the following, the Joule heating power in a UD generated by the magnetic field gradient is calculated for three magnetic fields defined by relations (1a), (1b) and (1c). For the electric current density, $\boldsymbol{J}$, we have (cgs)

$\boldsymbol{J}=\frac{c}{4 \pi} \boldsymbol{\nabla} \times \boldsymbol{B}=-\frac{c}{4 \pi} \frac{\partial B}{\partial r} \hat{a}_{\varphi}$ 
where $\hat{a}_{\varphi}$ is the azimuthal unit vector in cylindrical coordinate. For the three magnetic field functions we have:

a)

$$
\left|J_{\mathrm{a}}\right|=\frac{c \gamma B_{0}}{4 \pi} \frac{n}{R-U}\left(\frac{R-r}{R-U}\right)
$$

b)

$$
\left|\boldsymbol{J}_{\mathrm{b}}\right|=\frac{c \gamma B_{0}}{4 \pi} \frac{\alpha}{r\left(1+(r / U)^{\alpha}\right)^{2}}\left(\frac{r}{U}\right)^{\alpha},
$$

c)

$$
\left|\boldsymbol{J}_{\mathrm{c}}\right|=\frac{c \gamma B_{0}}{4 \pi} \frac{2 r}{U^{2}} \exp \left(-\frac{r^{2}}{U^{2}}\right) \text {. }
$$

Now, the Joule heating power, $Q$ (cgs), in unit length of the UD column can be calculated from

$Q=\frac{4 \pi}{c^{2}} \int_{0}^{\infty} \eta J^{2}(2 \pi r \mathrm{~d} r)$.

For the current density (14a), the mean Joule heating power can be evaluated as

$Q_{\mathrm{a}}=\frac{\eta}{2} \gamma^{2} B_{0}^{2} \frac{n^{2}}{(R-U)^{2 n}}\left[\int_{U}^{R}(R-U)^{2 n-2} r \mathrm{~d} r\right]$,

by a simple integration,

$Q_{\mathrm{a}}=4 \pi \eta u_{\gamma B} \frac{n^{2}}{(R-U)^{2 n}}\left[\frac{U(R-U)^{2 n-1}}{2 n-1}+\frac{(R-U)^{2 n}}{2 n(2 n-1)}\right]$.

Here and in the following relations it is assumed that $u_{\gamma B}=$ $\frac{\gamma^{2} B_{0}^{2}}{8 \pi}$. For $R$ close to $U$ (thin current sheet), $Q_{\mathrm{a}}$ is simplified to

$Q_{\mathrm{a}} \approx 4 \pi \eta u_{\gamma B}\left(\frac{U}{R-U} \frac{n^{2}}{2 n-1}\right)$.

For the case of (14b)

$Q_{\mathrm{b}}=\frac{\eta}{2} \gamma^{2} B_{0}^{2} \frac{\alpha^{2}}{U^{2}}\left[\int_{0}^{\infty} \frac{(r / U)^{2 \alpha-2}}{\left(1+(r / U)^{\alpha}\right)^{4}} r \mathrm{~d} r\right]$,

by partial integration, the integral in the bracket is evaluated easily as $\left[\frac{U^{2}}{6 \alpha}\right]$. Then we have

$Q_{\mathrm{b}}=4 \pi \eta u_{\gamma B}\left(\frac{\alpha}{6}\right)$.

For the case of (14c),

$Q_{\mathrm{c}}=\frac{\eta}{2} \gamma^{2} B_{0}^{2} \frac{4}{U^{4}}\left[\int_{0}^{\infty} r^{3} \exp \left(\frac{2 r^{2}}{U^{2}}\right) \mathrm{d} r\right]$,

the value of the bracket can be calculated easily as $\left[\frac{U^{4}}{8}\right]$. then

$Q_{\mathrm{c}}=4 \pi \eta u_{\gamma B}\left(\frac{1}{2}\right)$

One can see that the obtained Joule heating powers in Eqs. (17), (18) and (19) are proportional to

$P=4 \pi \eta u_{\gamma B}$.

The current sheet generated from the magnetic field of the forms (1a) and (1b) becomes thinner with larger $n$ and $\alpha$, respectively. In $B_{\mathrm{a}}(r)$ (see Fig. 1), for larger $n$ the smoothness at the maximum becomes wider and the gradient becomes larger. This is because the magnetic field and the current density for larger $n$ reach their maximum more quickly and the positions of these maximums are closer to $U$. Therefore, for large $n$ value,
Table 1. Estimates for Joule heating power $P$.

\begin{tabular}{ccccccc}
\hline \hline & & & & $P$ & $(\mathrm{cgs})$ & \\
& & & ---- & ---- & ---- & ---- \\
$\tau$ & $\sigma(\mathrm{cgs})$ & $\eta(\mathrm{cgs})$ & $\gamma=0.1$ & 0.2 & 0.5 & 1.0 \\
\hline 0.01 & $10^{9}$ & $10^{11}$ & $4.5 \times 10^{15}$ & $1.8 \times 10^{16}$ & $1.1 \times 10^{17}$ & $4.5 \times 10^{17}$ \\
0.1 & $10^{10}$ & $10^{10}$ & $4.5 \times 10^{14}$ & $1.8 \times 10^{15}$ & $1.1 \times 10^{16}$ & $4.5 \times 10^{16}$ \\
1.0 & $10^{11}$ & $10^{9}$ & $4.5 \times 10^{13}$ & $1.8 \times 10^{14}$ & $1.1 \times 10^{15}$ & $4.5 \times 10^{15}$ \\
\hline
\end{tabular}

and/or $R$ close to $U$, the thinness coefficient, $\xi=\left(\frac{U}{R-U} \frac{n^{2}}{2 n-1}\right)$ in Eq. (17) can be much larger than 1 . In $B_{\mathrm{b}}(r)$, the thickness of the current sheet decreases by increasing $\alpha$. Also for large $\alpha$, the thinness coefficient $\xi=\left(\frac{\alpha}{6}\right)$ in Eq. (18) can be much larger than 1 . If the boundary of the UD column is sharp the thinness coefficient will be very large. However, because of the low resolution of the magnetic field measurements in UDs, there is no observational evidence which gives us the correct form of the magnetic field in the UDs.

\section{The effect of Joule heating on UD brightness}

Here, some estimates are given for the values of the Joule heating power and the rate of the Joule dissipation energy in a UD on the basis of Eq. (20). For $\sigma$, the electrical conductivity, we use $\sigma_{33}$, defined by Oster (1968) in terms of the components of electrical conductivity matrix, to take into account a possible anisotropy. He has computed this quantity for Van't Veer's (1963) sunspot model. He showed that $\sigma_{33}$ varies from about $10^{9}(\mathrm{cgs})$ at $\tau \sim 10^{-2}$ to about $10^{12}(\mathrm{cgs})$ at $\tau \sim 10$. If we accept these values for electrical conductivity, $\sigma$, in the umbra, then the resistivity, $\eta$, can be calculated from $\eta=\frac{c^{2}}{4 \pi \sigma}$ (cgs). The values of $\sigma$ and $\eta$ at the three different optical depths, $\tau$, are given in Table 1 . Table 1 also lists some estimates for $P\left(\mathrm{erg} \mathrm{cm}^{-1} \mathrm{~s}^{-1}\right)$ at these optical depths and for four different $\gamma$ values. In these estimates we assumed $B_{0}=3000 \mathrm{G}$.

The mean rate of Joule dissipation energy, $E_{\mathrm{J}}\left(\mathrm{erg} \mathrm{s}^{-1}\right)$, within the UD column is obtained from

$E_{\mathrm{J}}=\bar{\xi} \int_{\mathrm{UD} \text { Column }} P \mathrm{~d} z=\bar{\xi} \bar{P} H$,

where $\bar{\xi}$ is the mean thinness coefficient of the current sheet, $\bar{P}$ is the average of $P$ along the UD column and $H$ is a typical height (length) of a UD column.

If it is assumed that the temperature of the gas inside the UD column is (at least) the same as temperature of the surrounding umbra - because of high radiative dissipation (see Sect. 2), and without considering Joule heating - the heat generated from Joule heating should explain the extra brightness of the UD compared to its surroundings. Thus, for the mean energy flux radiated from the UD, $F_{\mathrm{UD}}\left(\mathrm{erg} \mathrm{cm}^{-2} \mathrm{~s}^{-1}\right)$, we can write

$F_{\mathrm{UD}}=F_{\mathrm{u}}+(\beta \bar{\xi}) \frac{\bar{P} H}{A_{\mathrm{UD}}}$,

where, $F_{\mathrm{u}}\left(\mathrm{erg} \mathrm{cm}^{-2} \mathrm{~s}^{-1}\right)$ is the mean energy flux emitted from the surrounding umbra. $A_{\mathrm{UD}}$ is the surface area of a typical UD, 
Table 2. Estimates for excess temperature and brightness of UDs relative to their surrounding, resulting from only Joule heating.

\begin{tabular}{ccccccccccc}
\hline \hline & & \multicolumn{3}{c}{$\beta \bar{\xi}=1$} & \multicolumn{1}{c}{10} & \multicolumn{3}{c}{50} \\
& & ---- & ---- & ---- & ---- & -------- & ---- & -------- \\
$\gamma$ & $\bar{P}(\mathrm{cgs})$ & $\Delta F_{\mathrm{UD}}^{(*)}$ & $\frac{F_{\mathrm{UD}}}{F_{\mathrm{u}}}$ & $\Delta T_{\mathrm{UD}}(\mathrm{K})$ & $\Delta F_{\mathrm{UD}}^{(*)}$ & $\frac{F_{\mathrm{UD}}}{F_{\mathrm{u}}}$ & $\Delta T_{\mathrm{UD}}(\mathrm{K})$ & $\Delta F_{\mathrm{UD}}^{(*)}$ & $\frac{F_{\mathrm{UD}}}{F_{\mathrm{u}}}$ & $\Delta T_{\mathrm{UD}}(\mathrm{K})$ \\
\hline 0.1 & $4.5 \times 10^{13}$ & $4.5 \times 10^{6}$ & $\sim 1$ & 0 & $4.5 \times 10^{7}$ & $\sim 1$ & 3 & $2.2 \times 10^{8}$ & 1.01 & 14 \\
0.2 & $1.8 \times 10^{14}$ & $1.8 \times 10^{7}$ & $\sim 1$ & 1 & $1.8 \times 10^{8}$ & 1.01 & 12 & $9 \times 10^{8}$ & 1.06 & 60 \\
0.5 & $1.1 \times 10^{15}$ & $1.1 \times 10^{8}$ & $\sim 1$ & 8 & $1.1 \times 10^{9}$ & 1.07 & 73 & $5.5 \times 10^{9}$ & 1.38 & 334 \\
1.0 & $4.5 \times 10^{15}$ & $4.5 \times 10^{8}$ & 1.03 & 31 & $4.5 \times 10^{9}$ & 1.31 & 228 & $2.2 \times 10^{10}$ & 2.52 & 1037 \\
\hline
\end{tabular}

(*) $\Delta F_{\mathrm{UD}}=F_{\mathrm{UD}}-F_{\mathrm{u}},\left(\mathrm{erg} \mathrm{cm}^{-2} \mathrm{~s}^{-1}\right)$.

$\beta(<1)$ is the fraction of the total energy which reaches the surface of UD and is radiated from it. In the other word, $\frac{A_{\mathrm{UD}}}{\beta}$ is the mean total area of the UD column from which the energy is radiated. Parameters $\bar{\xi}(>1), \bar{P}$ and $H$ have the same definitions as in Eq. (21).

The UD column probably does not extend very high. Its intensity and magnetic field strength are very small in the layer where the spectral lines are formed. It seems that the temperature difference between a UD and its surrounding umbra, disappears at $z=100 \mathrm{~km}$ above $\tau \sim 1$ level, namely at $\tau \sim 0.2$ (Socas Navarro 2002). Thus, it is reasonable that the value of $P$ at $\tau \sim 1$ (see Table 1 ) is considered as a $\bar{P}$ value along the UD column. Also, the mean length of the UD column may be estimated at about $H=300 \mathrm{~km}$, (see Wiehr 1994 and Lites et al. 1991). These estimates have been used in Table 2.

The energy flux of the umbra, $F_{\mathrm{u}}$, with a temperature typically, $T_{\mathrm{u}}=4000 \mathrm{~K}$ may be obtained from the StefanBoltzmann relation as

$F_{\mathrm{u}}=\sigma_{\mathrm{SB}} T_{\mathrm{u}}^{4}=1.45 \times 10^{10} \mathrm{erg} \mathrm{cm}^{-2} \mathrm{~s}^{-1}$,

where $\sigma_{\mathrm{SB}}=5.67 \times 10^{-5} \mathrm{erg} \mathrm{cm}^{-2} \mathrm{~K}^{-4} \mathrm{~s}^{-1}$, is the Stefan-Boltzmann constant.

For computing the second term in Eq. (22), $\Delta F_{\mathrm{UD}}=F_{\mathrm{UD}}-$ $F_{\mathrm{u}}$, it is assumed that $A_{\mathrm{UD}} \approx 3 \times 10^{14} \mathrm{~cm}^{2}$ (a UD with mean diameter of $200 \mathrm{~km}$ ). Some estimates for this term have been calculated by selecting different values for $\gamma$ and $(\beta \bar{\xi})$ which have been given in Table 2.

From the relation $F_{\mathrm{UD}}=\sigma_{\mathrm{SB}} T_{\mathrm{UD}}^{4}$, we can evaluate the apparent UD temperature, $T_{\mathrm{UD}}$, and the apparent difference of the UD temperature relative to the surrounding umbra, $\Delta T_{\mathrm{UD}}$. These results have been summarized in Table 2 . We can see that Joule heating can explain the brightness of the UDs when the gradient of the magnetic field at the boundary of the UD column is sharp and/or the field reduction in the UD column is considerable (large $\gamma$ value $(\leq 1)$ ). Recently, Tritschler $\&$ Schmidt (2002) reported an average temperature difference of $330 \mathrm{~K}$ between the UDs and their surrounding umbra. This is consistent with temperature values in Table 2 for $\gamma=1, \beta \bar{\xi}=10$ and $\gamma=0.5, \beta \bar{\xi}=50$.

\section{Conclusions}

In this paper a UD model has been introduced which needs neither significant upward mass motion nor energy transported by radiation from the bottom of the UD column. The brightness of the UDs (at least a kind of UDs) can be explained by it. The variability of the density, intensity and upward plasma motion in the UDs suggests that the UDs do not result from regular convective modes in the umbra (Gokhale \& Zwaan 1972). It seems that the UDs (at least a group of them) are secondary effects of small and temporary perturbations imposed locally on the umbral equilibrium state. Thus, the global stability conditions governing on the umbra try to omit these small disturbances - regions (columns) with reduced density and magnetic field strength as the "seed" of the UDs. Thereby, plasma falls laterally down the resulting (UD) column, the column collapses, and some plasma escapes from it.

On the basis of the estimates given in Sect. 3, the outflow in the UD varies from zero at the bottom of the UD column to a few hundred $\mathrm{km} \mathrm{s}^{-1}$ in the upper layers (heights). This result is in agreement with the observations by Lites et al. (1991). Also, it is in agreement with Pahlke \& Wiehr (1990) who inferred that outflow within the UDs accelerates with height.

It seems that if the pressure gradient around the column is small - as a result of lower $\gamma$ value or/and smaller $B_{0}-$ the rate of radius reduction will be small. In such situation, a UD lives longer and the mass upward motion in it is slow, and vice versa.

According to the results obtained in Sect. 5, without considering the amount of the hot gas inflow from below, the UD brightness depends directly on three factors, a) the magnitude of the undisturbed field of its surroundings, $B_{0}$, b) the value of the field reduction fraction, $\gamma$, in the UD, and c) the magnetic field gradient around the UD column or the thinness coefficient. Therefore, the brightness of the UDs which are created in a certain region of the umbra ( $B_{0}$ is a constant for all of them), depends on factors (b) and (c). Thus, the presented model predicts that - if injection of hot gas from the bottom of the UD column is not continued - the brightest UDs which live in a certain region of umbra, probably have $\gamma$ values close to one (very weak magnetic field inside UD column), shorter lifetime, and larger outflow. Probably, for the long-lived UDs, a gas inflow is needed from the bottom of the UD column for a time interval. This also may affect the upward gas motion and the temperature of a UD.

Acknowledgements. I am much indebted to Prof. Jalal Samimi for his invaluable support. Also I am very grateful to Michal Sobotka and an anonymous referee for their constructive comments. 


\section{References}

Adjabshirzadeh, A., \& Koutchmy, S. 1983, A\&A, 122, 1

Beckers, J. M., \& Schröter, E. H. 1968, Sol. Phys., 4, 303

Bumba, V., \& Suda, J. 1980, Bull. str. Inst. Czechoslovaki, 31, 101

Buurman, J. 1973, A\&A, 29, 329

Choudhuri, A. R. 1986, ApJ, 302, 809

Degenhardt, D., \& Lites, B. W. 1993, ApJ, 404, 383

Ewell, M. W. 1992, Sol. Phys., 137, 215

Gokhale, M. H., \& Zwaan, C. 1972, Sol. Phys., 26, 52

Grossmann-Doerth, U., Schmidt, W., \& Schröter, E. H. 1986, A\&A, 156,347

Hirayama, H. 1992, Sol. Phys., 137, 33

Kneer, F., Soltau, D., \& Wiehr, E. 1990, in IAU Symp. 142, Basic Plasma Processures in the Sun, ed. E. R. Priest, \& V. Krishan (Dordrecht: Kluwer), 113

Knobloch, E., \& Weiss, N. O. 1984, MNRAS, 207, 203

Koutchmy, S., \& Adjabshirzadeh, A. 1981, A\&A, 99, 111

Kusoffsky, V., \& Lundstedt, H. 1986, A\&A, 160, 51

Lites, B. W., Bida, T. A., Johannesson, A., \& charmer, G. B. 1991, ApJ, 373, 683
Maltby, P., Avrett, E. H., Corlsson, M., et al. 1986, ApJ, 306, 284

Oster, L. 1968, Sol. Phys., 3, 543

Pahlke, K.-D., \& Wiehr, E. 1990, A\&A, 228, 246

Parker, E. N. 1979a, ApJ, 230, 905

Parker, E. N. 1979b, ApJ, 234, 333

Petrovay, K., Martinez Pillet, V., \& van driel-Gesztelyi, L. 1999, Sol. Phys., 188, 315

Rimmele, T. R. 1997, ApJ, 490, 458

Sobotka, M., Bonet, J. A., \& Vazquez, M. 1992b, A\&A, 260, 437

Sobotka, M., Bonet, J. A., \& Vazquez, M. 1992a, A\&A, 257, 757

Sobotka, M., Bonet, J. A., \& Vazquez, M. 1993, ApJ, 415, 832

Sobotka, M., Brandt, P. N., \& Simon, G. 1997a, A\&A, 328, 682

Sobotka, M., Brandt, P. N., \& Simon, G. 1997b, A\&A, 328, 689

Socas Navarro, H. 2002, in Current Theoretical Models and Future High Rsolution Solar Observations: Preparing for ATST, ed. A. A. Pevtsov, \& H. Uitenbroek, ASP Conf. Ser., in Press

Tritschler, A., \& Schmidt, W. 2002, A\&A, 388, 1048

Van't Veer, F. 1963, Ann. Astrophys., 26, 185

Wiehr, E. 1994, A\&A, 287, 1

Wiehr, E., \& Degenhard, D. 1993, A\&A, 278, 584

Zhao, J., \& Kosovichev, A. G. 2001, ApJ, 557, 384 